[0212-7199 (2007) 24: 10; pp 494-496] ANALES DE MEDICINA INTERNA Copyright (C) 2007 ARAN EDICIONES, S.L.

AN. MED. InTERnA (Madrid) Vol. 24, N. ${ }^{\circ} 10$, pp. 494-496, 2007

\title{
RS3PE asociado a tuberculosis
}

\section{F. J. NICOLÁS-SÁNCHEZ, J. R. ROZADILLA SACANELL, A. GORT OROMÍ, G. TORRES CORTADA, T. SOLER ROSELL, R. M. SARRAT-NUEVO, M.E. NICOLÁS-SÁNCHEZ, J. CABAU-RUBIES}

Servicio de Medicina Interna. Hospital de Santa María. Lleida

\author{
RS3PE ASSOCIATED WITH TUBERCULOSIS
}

\section{RESUMEN}

El síndrome de sinovitis simétrica seronegativa remitente del anciano con edema y fóvea (remitting seronegative symmetrical synovitis with pitting edema) (RS3PE), se caracteriza por la aparición de una poliartritis simétrica bilateral seronegativa asociada a edema con fóvea en el dorso de las manos, y factor reumatoide negativo. Su asociación a tuberculosis no se había descrito previamente. Se presenta el caso de un paciente de 89 años que consultó por anorexia de mes y medio de evolución acompañada de edema en manos y pies, con factor reumatoide negativo. Presentaba un infiltrado pulmonar, cuyo estudio microbiológico reveló la infección por Mycobacterium tuberculosis.

PALABRAS CLAVE: RS3PE. Sinovitis simétrica seronegativa remitente del anciano con edema y fóvea. Tuberculosis.

\begin{abstract}
The remitting seronegative symmetrical synovitis with pitting edema of the elderly patient with edema and fovea (RS3PE), characterizes for the appearance of one polyarthritis symmetrical with fovea in the back of the hands, and negative reumatoideal factor. The association to tuberculosis had not been described before. One presents the case of a 89-yearold patient who consulted for anorexia of month and a half of evolution accompanied of edema in hands and feet, with negative reumatoideal factor. He was presenting a pulmonary infiltrated, which microbiological study revealed the infection for Mycobacterium tuberculosis.
\end{abstract}

KEY WORDS: RS3PE. Remitting seronegative symmetrical synovitis with pitting edema. Tuberculosis.

Nicolás-Sánchez FJ, Rozadilla Sacanell JR, Gort Oromí A, Torres Cortada G, Soler Rosell T, Sarrat-Nuevo RM, Nicolás-Sánchez ME, CabauRubies J. RS3PE asociado a tuberculosis. An Med Interna (Madrid) 2007; 24: 494-496.

\section{INTRODUCCIÓN}

El síndrome RS3PE (remitting seronegative symmetrical synovitis with pitting edema) descrito por McCarty en 1985 (1), se caracteriza por la aparición de una poliartritis simétrica bilateral seronegativa asociada a edema con fóvea en el dorso de las manos. También se conoce como polisinovitis aguda edematosa del anciano, su etiología es desconocida y tiene buen pronóstico. Su presencia también se ha relacionado con enfermedades infecciosas (1-6), sistémicas, autoinmunitarias, tumores sólidos, y neoplasias hematológicas (7-9). Su asociación a tuberculosis no se había descrito previamente.

\section{CASO APORTADO}

Paciente varón de 89 años sin antecedentes patológicos de interés que consultó por anorexia de mes y medio de evolución acompañada de edema en manos y pies. Refería algún episodio de rectorragia. La exploración física solo reveló el edema con fóvea limitado a articulaciones metacarpofalángicas y metatarso falángicas (Fig. 1). Con el diagnóstico de sospecha de RS3PE se inició terapia con prednisona $20 \mathrm{mg} / 24$ horas, desapareciendo el edema completamente a los 5 días de tratamiento (Fig. 2). Dado que este que este síndrome puede aparecer asociado a otras patologías se realizó el estudio del paciente.

En las pruebas complementarias la hemoglobina era de $\mathrm{Hb} 10,3$ $\mathrm{g} / \mathrm{dL}$, con VCM 94,5 fl. La bioquímica la glucosa, urea, creatinina, ácido úrico, calcio total, fosfato, colesterol, colesterol-HDL, colesterol-LDL, proteínas totales, GPT, LDH, GGT, Na, K eran normales. Cobalaminas y folatos normales. Beta-2-microglobulina normal. En el proteinograma apareció una banda monoclonal a IgA lambda. La proteína $\mathrm{C}$ reactiva y la VSG eran normales. La interleucina 6 fue normal. Serología de VIH negativa. El factor reumatoide, los anticuerpos antinucleares y los anticuerpos anticitoplasma neutrófilo fueron negativos. El PSA fue normal. El fenotipo HLA-B27 fue positivo. La proteinuria de Bence-Jones fue negativa. La colonoscopia fue normal. Las radiografías de manos y pies no mostraban lesiones erosivas. En la TAC torácica en el segmento posterior del LSD se apreciaba un infiltrado pulmonar que adoptaba una morfología ramificada (tree inbud) y nodular; en el LM también se observaba un infiltrado de morfología ramificada, nodular y una condensación subsegmentaria con broncograma aéreo; tractos pleuroparenquimatosos biapicales bronquiectasias cilíndricas de pared fina en LII y con

Trabajo aceptado: 28 de mayo de 2007

Correspondencia: Francisco José Nicolás Sánchez. Servicio de Medicina Interna. Hospital de Santa María. Avda. Alcalde Rovira Roure, 44. 25006 Lleida. e-mail: fnicolas@comll.cat 


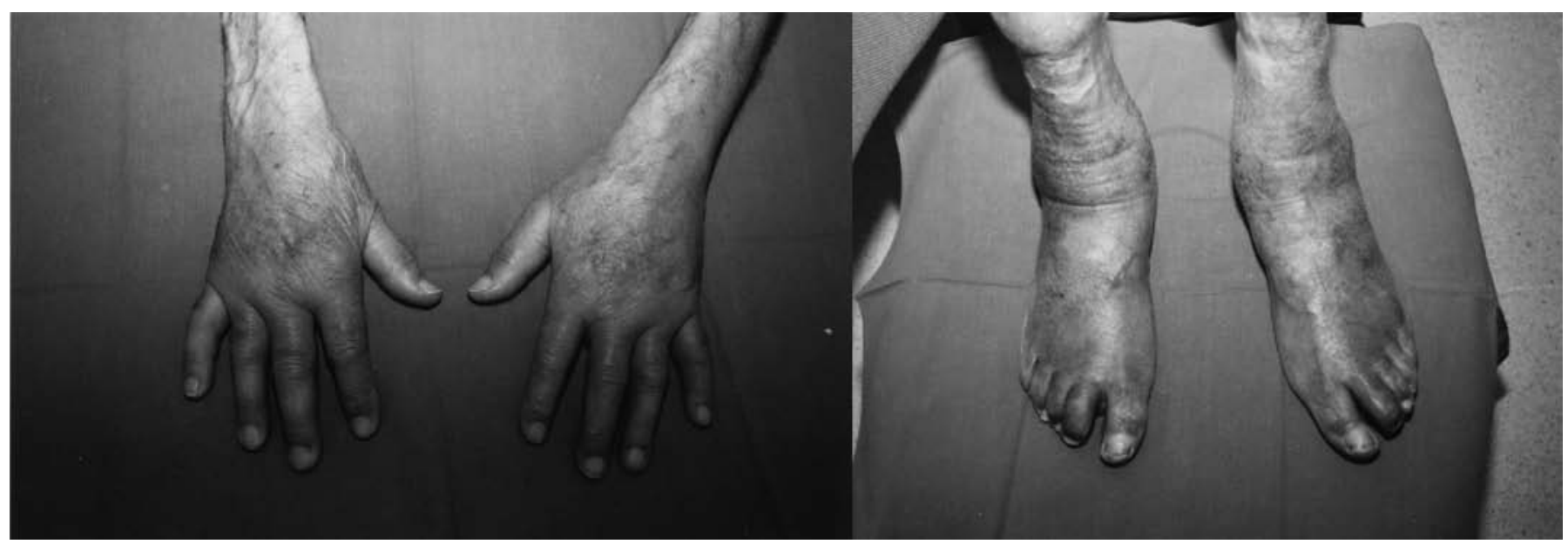

Fig. 1. Edema de manos y pies.

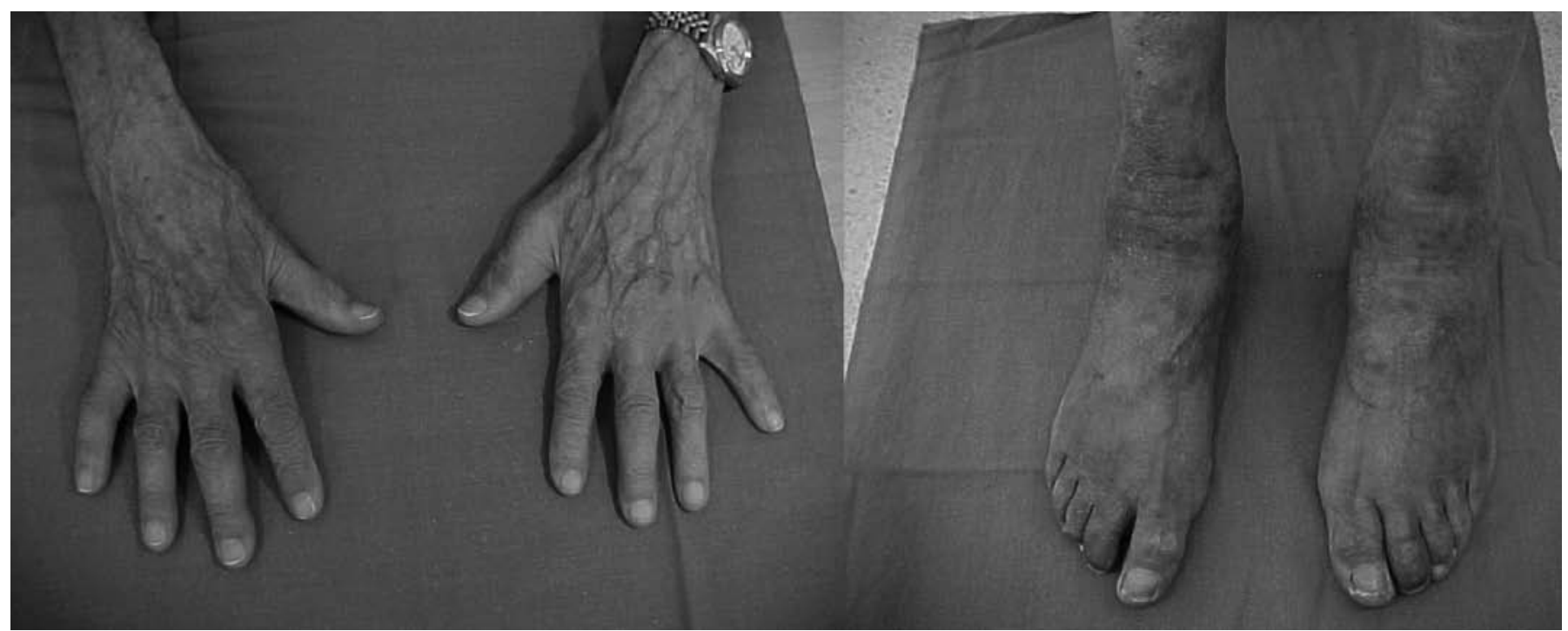

Fig. 2. Se observa la desaparición del edema tras 5 días de tratamiento corticoideo.

engrosamiento de língula, y pleura. TAC abdominal vejiga urinaria con engrosamiento del suelo vesical, con múltiples divertículos, hipertrofia prostática. La broncoscopia presentó una tráquea, y árbol bronquial izquierdo con signos inflamatorios crónicos, destacan divertículos y placas antracosis en todo el árbol bronquial; a nivel del árbol bronquial derecho, en bronquio intermediario unos $\mathrm{mm}$ antes de llegar a la entrada LM se observa infiltración mucosa y a la entrada de LM había una masa de aspecto necrótico y muy friable que obstruía totalmente la entrada, no se realizó biopsia bronquial por sangrado. El estudio citológico del BAS reveló un frotis inflamatorio agudo. El cultivo de Löwestein del BAS fue positivo para Mycobacterium tuberculosis.

En la evolución del paciente se observó que a los 5 días de iniciar el tratamiento corticoideo desapareció el edema de manos y pies, al mes se normalizó la hemoglobina. A los 4 meses de iniciar la prednisona se identificó al Mycobacterium tuberculosis pautándose isoniacida, rifampicina y pirazinamida, y suspendiendo el tratamiento corticoideo por la sospecha de enfermedad de Poncet. A los 3 meses de iniciar los tuberculostáticos y de retirar los corticoides reaparicieron el edema en manos y pies, por lo que se reintrodujo el tratamiento corticoideo.

\section{DISCUSIÓN}

El RS3PE aparece generalmente en varones mayores de 60 años, de forma brusca y con menos de un mes de evolución. Su etiología es desconocida y puede aparecer como un síndrome con entidad propia. En algunos trabajos se ha relacionado con HLA B27, pero no se ha podido confirmar su relación (10). Pero su aparición también se ha relacionado con la presencia de agentes infecciosos como la instilación vesical del bacilo de Calmette-Guerin, la enfermedad de Wiple, el parvovirus B19, la lepra, Mycoplasma pneumoniae (2-6); enfermedades sistémicas como la artritis reumatoide, la sarcoidosis, la polimialgia reumática o la arteritis de células gigantes, espondiloartropatías, artritis microcristalinas, el síndrome de Sjögren, artritis psoriásicas; factores genéticos; o también ser la primera manifestación de un síndrome paraneoplásico asociado a tumores sólidos como el carcinoma renal, prostático, gástrico, colon, y páncreas entre otros; neoplasias hematológicas como el linfoma no Hodgking, el mieloma múltiple o la leucemia linfoide crónica. 
La forma de presentación más frecuente es la de una poliartritis aguda que afecta a las articulaciones metacarpofalángicas e interfalángicas proximales con dolor y limitación funcional, acompañada de edema con fóvea en el dorso de la manos. También se pueden afectar las articulaciones de hombros, codos, muñecas, caderas, tobillos y pies. El edema se produce por un aumento de la permeabilidad capilar en tejido celular subcutáneo y tendones de los músculos extensores. El caso descrito presentaba poliartritis de manos y pies acompañado de edema dorso de las manos y pies con fóvea. No suelen presentar síndrome tóxico, pero en este caso probablemente se podría atribuir a la enfermedad tuberculosa.

En algunos casos se observa una elevación de VSG y PCR que en este paciente no se evidenció.

Con el tratamiento corticoideo (prednisona dosis 10-20

\section{Bibliografía}

1. Mc Arthy DJ, O' Duffy JD, Pearson L, Hunter JB. Remitting seronegative symmetrical synovitis with pitting edema. RS3PE syndrome. JAMA 1985; 254: 2763-7.

2. Ramos Soria F, Doménech Santasusana M, Guasch Jordan I, López Borrás E, Busquet Solé N. Síndrome RS3PE como primera manifestación de un carcinoma prostático. An Med Interna (Madrid) 2003; 20: 217-8.

3. El Mahou S, Popa L, Constantin A, Cantagrel A, Aberrane A, Jamard B, Mazieres B, Laroche M. Remitting seronegative symmetrical synovitis pitting oedema after BCG instillation. Clin Rheumatol 2006; 25: 566-7. Epub 2006 Mar 25.

4. Matsuda M, Shimojima Y, Gono T, Ishii W, Kaneko K, Yazaki M, Ikeda SIRemitting seronegative symmetrical synovitis with pitting oede$\mathrm{ma}$ /polymyalgia rheumatica after infection with Mycoplasma pneumoniae. Ann Rheum Dis 2005; 64: 1797-8.

5. Helling CA, Locursio A, Manzur ME, Sormani de Fonseca ML. Remitting seronegative symmetrical synovitis with pitting edema in leprosy. Clin Rheumatol 2006; 25: 95-7. Epub 2005 Sep 21.

6. Perandones CE, Colmegna I, Arana RM. Parvovirus B19: Another $\mathrm{mg}$ /día) a dosis bajas remite de forma completa en pocos días (11). En este caso se inició tratamiento con prednisona $20 \mathrm{mg}$ cada 24 horas, observándose resolución total del edema de manos y pies a los 5 días de iniciar el tratamiento. La reaparición del edema de manos y pies a los 3 meses de retirar los corticoides y de haber iniciado el tratamiento tuberculostático, hace improbable el que pueda tratarse de la enfermedad de Poncet (12). Los casos secundarios a neoplasias a veces no responden al tratamiento corticoideo, pero si lo hacen con la curación del tumor. Cuando el RS3PE ha sido la manifestación de un síndrome paraneoplásico su reaparición puede indicar la recidiva del tumor.

El pronóstico es bueno cuando se trata de un síndrome aislado, pero siempre hay que tener presente de que puede asociarse a otras patologías. agent associated with remitting seronegative symmetrical synovitis with pitting edema. J Rheumatol 2005; 32: 389-90.

7. Torres A, Cuende E, De Pablos M, Lezaun MJ, Michaus L, Vesga JC. Remitting seronegative symmetrical synovitis with pitting edema associated with subcutaneous Streptobacillus moniliformis abscess. J Rheumatol 2001; 28: 1696-8.

8. Bruscas Izu C, Medrano San Ildefonso M, Simon L. RS3PE syndrome: Eeport of 11 cases. An Med Interna 2000; 17: 485-7.

9. Parra Rodenas JV, Calvo Catala J, González-Cruz Cervellera MI, Cervera Moscardo J, Valero Prieto I. RS3PE syndrome: Presentation of two cases. An Med Interna (Madrid) 1996; 13: 601-2.

10. Pease C, Bhakta B. RS3PE syndrome: The relation with HLA B27. Ann Rheum Dis 2000; 59: 239-40.

11. Paira S, Graf C, Roverano S, Rossini J. Remitting seronegative symmetrical synovitis with pitting oedema: study of 12 cases. Clin Rheumatol 2002; 21: 146-9.

12. Dall L, Long L, Standford J. Poncet's disease: Tuberculous rheumatism. Rev Infec Dis 1989; 11: 105-7. 